\title{
Larval habitat segregation between the molecular forms of the mosquito Anopheles gambiae in a rice field area of Burkina Faso, West Africa
}

\author{
G. G I M O N NEA U ${ }^{1}$, M. P O M B I ${ }^{2}$, M. C H O I S Y ${ }^{1}$, S. M O R A N D \\ R. K. D A B I R É ${ }^{4}$ and F. S I M A R D ${ }^{1,4}$ \\ ${ }^{1}$ MIVEGEC (Maladies Infectieuses et Vecteurs: Ecologie, Genetique, Evolution et Contrôle), UMR224, Institut de Recherche \\ pour le Développement (IRD), Montpellier, France, ${ }^{2}$ Sezione di Parassitologia, Dipartimento di Sanità Pubblica e Malattie \\ Infettive, Università di Roma 'Sapienza', Rome, Italy, ${ }^{3}$ Institut des Sciences de l'Evolution de Montpellier (ISEM), UMR 5554, \\ Université de Montpellier, Montpellier, France and ${ }^{4}$ Institut de Recherche en Sciences de la Santé (IRSS), Direction Régionale \\ de l'Ouest (DRO), Bobo Dioulasso, Burkina Faso
}

\begin{abstract}
In West Africa, lineage splitting between the $\mathrm{M}$ and $\mathrm{S}$ molecular forms of the major Afro-tropical malaria mosquito, Anopheles gambiae (Diptera: Culicidae), is thought to be driven by ecological divergence, occurring mainly at the larval stage. Here, we present evidence for habitat segregation between the two molecular forms in and around irrigated rice fields located within the humid savannahs of western Burkina Faso. Longitudinal sampling of adult mosquitoes emerging from a range of breeding sites distributed along a transect extending from the heart of the rice field area into the surrounding savannah was conducted from June to November 2009. Analysis revealed that the two molecular forms and their sibling species Anopheles arabiensis are not randomly distributed in the area. A major ecological gradient was extracted in relation to the perimeter of the rice fields. The $\mathrm{M}$ form was associated with larger breeding sites mostly consisting of rice paddies, whereas the $\mathrm{S}$ form and An. arabiensis were found to depend upon temporary, rain-filled breeding sites. These results support hypotheses about larval habitat segregation and confirm the suggestion that the forms have different larval habitat requirements. Segregation appears to be clearly linked to anthropogenic permanent habitats and the community structure they support.
\end{abstract}

Key words. Anopheles gambiae, community structure, hydro-periodicity, larval habitat, niche partitioning, segregation.

\section{Introduction}

Environmental changes by habitat modification often expose populations to new ecological constraints, including variations in climatic conditions, interspecies interactions, resource availability and other biotic and abiotic components of their respective ecosystems (Schluter, 2001). Divergent selection acts on species evolving under contrasting environments and may ultimately result in reproductive isolation through a process known as ecological speciation (Schluter, 2009). Although it has proven difficult to demonstrate ecological speciation as a byproduct of local adaptation in the field (Nosil et al., 2009), there are now multiple examples of rapid speciation arising from ecological diversification in the literature (Schluter, 2001, 2009; Mallet, 2008; Nosil et al., 2009) and it has received experimental support in various model organisms (Rice \& Hostert, 1993; Funk, 1998; Funk et al., 2002). Such a process may be prompted by

Correspondence: Geoffrey Gimonneau, Team BEES (Biology, Ecology and Evolution of Vector Systems), MIVEGEC, Institut de Recherche pour le Développement (IRD), UMR224, 911 Avenue Agropolis, BP 64501, 34394 Montpellier, France. Tel.: +33 (0) 4670432 22; Fax: +33 (0) 4675420 44; E-mail: geoffrey.gimonneau@gmail.com 
environmental modifications that modify ancestral habitats and offer new ecological niches to resident, as well as invasive, species.

Today, human activities are the main causes of disturbances in ecosystems that drive species towards adaptation or extinction (Palumbi, 2001). In West Africa, man-made environmental modifications are suspected to have instigated the diversification and spread of the major human malaria vector, the mosquito Anopheles gambiae sensu stricto, through the creation of new ecological niches in marginal habitats (Coluzzi et al., 1985; Coluzzi, 1999). The spread of new agricultural practices, combined with deforestation and the creation of water reserves, has been presumed to represent the initial environmental changes prompting ecological niche specialization within An. gambiae s.s. through divergent selection in these new habitats (Coluzzi et al., 2002). Understanding the distribution and dynamics of vector species in relation to fluctuations in their environments is of paramount importance if we are to better understand the epidemiology of the diseases these mosquitoes transmit, predict their evolution in the face of ongoing global environmental change and eventually improve their control (Fontenille \& Simard, 2004).

Anopheles gambiae s.s. is split into two molecular forms known as ' $\mathrm{M}$ ' and ' $\mathrm{S}$ ', which are morphologically identical and largely sympatric throughout West Africa (della Torre et al., 2001, 2005), but show widespread molecular differences throughout their genomes (Lawniczak et al., 2010; Neafsey et al., 2010; White et al., 2010). Although the two molecular forms share the same resources, such as vertebrate hosts and freshwater habitats, they have been shown to diverge in some of their biological and ecological requirements (Coluzzi et al., 1985; Touré et al., 1994; Lehmann \& Diabaté, 2008; Costantini et al., 2009; Simard et al., 2009). In the dry savannahs of West Africa, the $\mathrm{S}$ form preferentially breeds in temporary aquatic habitats and is found during the rainy season only, whereas the $\mathrm{M}$ form is present all year round, breeding in man-made permanent aquatic habitats (Costantini et al., 2009; Simard et al., 2009). Such differences in the spatial and temporal distributions of these vector mosquitoes have implications for the epidemiology of malaria transmission: in areas where irrigated agricultural management provides permanent breeding opportunities, malaria is potentially transmitted throughout the year. However, although this ecological segregation between breeding sites is generally accepted, a quantitative framework formalizing such observations is still lacking.

In order to better understand the ecological determinants of larval habitat segregation between the molecular forms of An. gambiae, we undertook a longitudinal entomological survey in an area of extensive rice cultivation in southwest Burkina Faso (West Africa), where both molecular forms of An. gambiae occur, together with their sibling species, Anopheles arabiensis. The main objectives of this study were: (a) to determine the spatial distribution of An. gambiae sensu lato mosquitoes along a lentic (i.e. still or slowmoving) freshwater gradient during the rainy season, and (b) to associate environmental parameters with mosquito occurrence in order to characterize the ecological factors explaining habitat segregation at a local geographical scale. Systematic sampling of adults emerging from a range of larval development sites allowed us to depict the distribution pattern of An. gambiae s.l. mosquitoes in and around the rice cultivation area and to identify relevant biotic and abiotic factors that shape the ecological niches of these taxa.

\section{Materials and methods}

Study site and sampling scheme

The study was conducted in the vicinity of Bama $\left(11^{\circ} 23^{\prime} \mathrm{N}\right.$, $04^{\circ} 24^{\prime} \mathrm{W}$ ), a village in the Kou Valley, situated $30 \mathrm{~km}$ north of Bobo Dioulasso in southwestern Burkina Faso (Fig. 1). The village is surrounded by 1200 ha of irrigated rice fields embedded within a typical Guinean savannah background. The rainy season in this area extends from May to October and yearly rainfall averages $1200 \mathrm{~mm}$ (Costantini et al., 2009). The M form of An. gambiae is present throughout the year in high densities in rice fields. In surrounding savannahs, the $\mathrm{S}$ form is typically found in great numbers during the rainy season, together with An. arabiensis (Diabaté et al., 2002; Baldet et al., 2003; Costantini et al., 2009; Lefèvre et al., 2009).

The sampling plan was conceived using maps of the study area constructed from road maps (1: 1000 000), low altitudinal aerial photographs at $0.8 \mathrm{~m}$ resolution (Wellens et al., 2008) and field observations. In order to explore the distribution and ecological niche requirements of both molecular forms of An. gambiae and An. arabiensis, we focused on adult mosquitoes emerging from breeding sites because these represent the outcome of larval life and reflect its specific selective pressures. Eighty breeding sites were chosen along a $15-\mathrm{km}$ east-west transect running from the core of the rice fields to the surrounding savannah. Among these, 15 breeding sites were located within the perimeter of the rice fields and 65 were located in surrounding savannah (Fig. 1). Each larval habitat was geo-referenced (Garmin GPS Map 60cx; Garmin International, Inc., Olathe, KS, U.S.A.) and sampled every other week from June to November 2009 until all temporary larval development sites had dried out, resulting in a total of 960 samplings (i.e. 80 water collections sampled 12 times each in the course of the study).

\section{Mosquito collection}

Emergence traps covering $1 \mathrm{~m}^{2}$ of ground surface were set out in larval breeding sites for $24 \mathrm{~h}$. Fifteen aquatic habitats were sampled daily during 1 week to cover the whole sampling area (i.e. 80 breeding sites). The same procedure was repeated every other week. Emerging mosquitoes were collected from the trap using Centers for Disease Control (CDC) backpack aspirators (Clark et al., 1994). Mosquitoes were sexed and identified morphologically in the field (Gillies \& De Meillon, 1968) and stored at $-20{ }^{\circ} \mathrm{C}$ in individual tubes until further use in the laboratory. All mosquitoes from the An. gambiae complex were identified to species and molecular form using a recently developed polymerase chain reaction (PCR) assay (Santolamazza et al., 2008). However, when sample sizes per trap were $>20$, only 20 randomly selected specimens were 

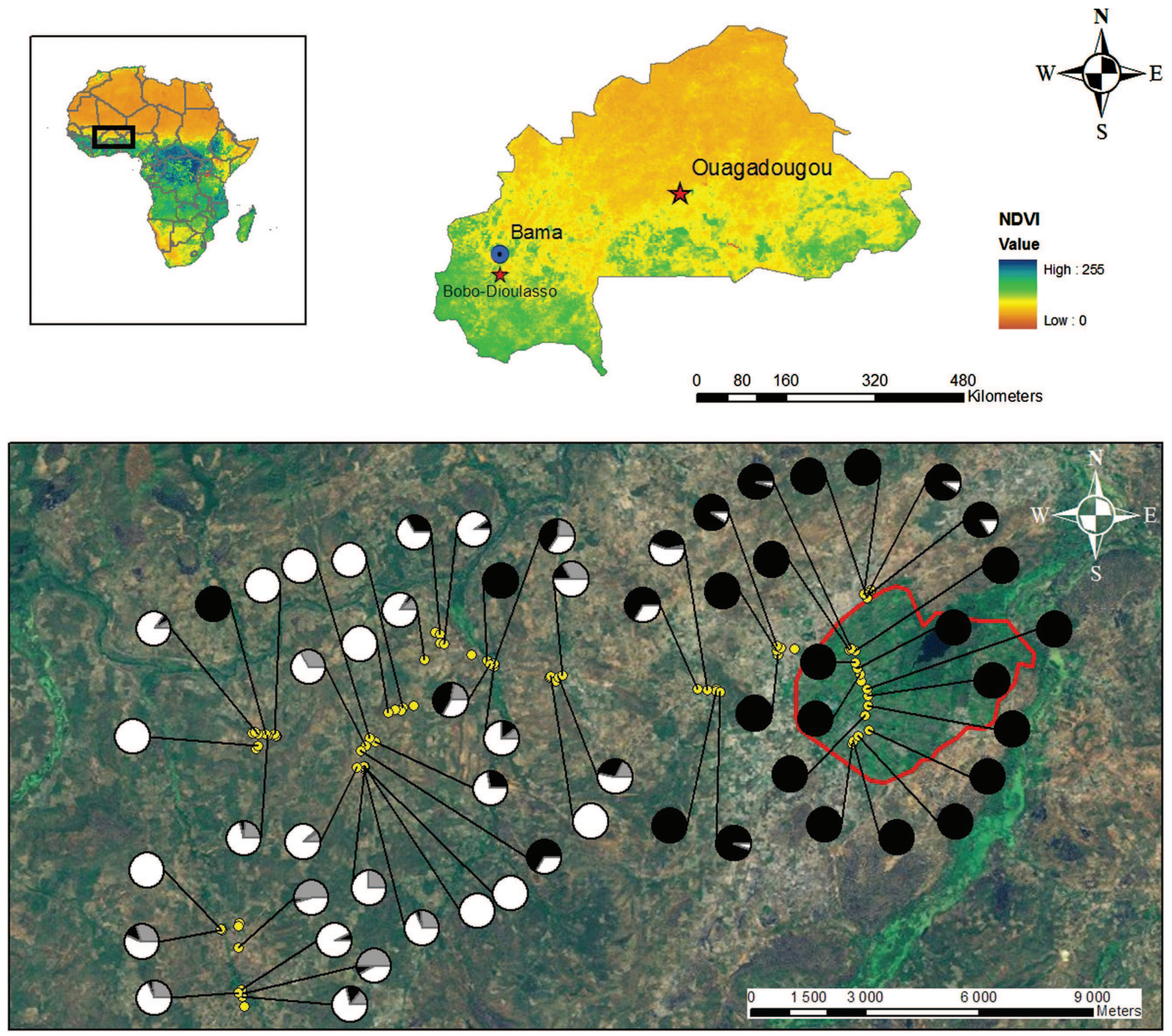

Fig. 1. Maps showing Burkina Faso in Africa, and Bama (blue dot) in Burkina Faso (Normalized Difference Vegetation Index; http://free.vgt.vito.be/index.php). A low-altitude aerial view (Wellens et al., 2008) shows the perimeter of the rice fields (red) and the 80 breeding sites (yellow dots) in Bama monitored throughout the 2009 rainy season. Pie charts show the relative frequencies and distributions of members of the Anopheles gambiae complex emerging from each larval development site (data pooled across collection dates). Black, $\mathrm{M}$ form; white, S form; grey, Anopheles arabiensis.

processed and the results were extrapolated to the remainder of the trap sample.

\section{Breeding site identification and recording of ecological parameters}

To record habitat type, each water collection was categorized as representing a collection from one of the following: stream pools, rice paddies, quarries, and road ruts. Nominal environmental variables (biotic and abiotic) used to characterize aquatic habitats were the presence of floating (mainly Marsilea polycarpa and Nymphea sp.) and emerging plants, algae, predators (mainly Notonectidae and Dytiscidae), Culex larvae [mainly Culex quinquefasciatus (Diptera: Culicidae)], floating debris, and turbidity (i.e. when the ground of the breeding site was not visible). General surroundings were characterized by three nominal variables: village, savannah, and culture. All these nominal variables were represented by dummy variables (Boolean) for subsequent analyses. These environmental factors were assessed visually and always by the same person for consistency. Continuous variables included the surface (in $\mathrm{m}^{2}$ ) and depth (in $\mathrm{cm}$ ) of the habitat and distances (in $\mathrm{m}$ ) to the nearest house and to the rice fields. The two former variables were assessed using a tape measure and the two latter variables were estimated using a geographic information system (ArcGis v9.3; Environmental Systems Research Institute, Inc.,

(C) 2011 The Authors

Medical and Veterinary Entomology (c) 2011 The Royal Entomological Society, Medical and Veterinary Entomology, doi: 10.1111/j.1365-2915.2011.00957.x 


\section{G. Gimonneau et al.}

Redlands, CA, U.S.A.). Collection month was represented by a series of dummy variables from June to November.

\section{Statistical analysis}

Statistical analysis was carried out for aquatic habitats at which at least one emerging adult of An. gambiae s.l. was collected. To explore whether the $\mathrm{M}$ and $\mathrm{S}$ forms of An. gambiae and their sibling species An. arabiensis were randomly distributed or ecologically structured in our study area, we performed a statistical randomization of the original occurrence data by simulating 5000 random matrices using the computer program EcoSim (Gotelli \& Entsminger, 2010). We used the C-score (Stone \& Roberts, 1990), Ecosim's default co-occurrence index that measures the average number of checkerboard units between all possible pairs of species in a co-occurrence matrix. We used EcoSim's recommended randomization algorithm, which maintains fixed sums for rows and fixed sums for columns so that each matrix generated includes the same number of species and samples as the original matrix (Connor \& Simberloff, 1979). If a community is ecologically structured, the C-score should be greater than that expected by chance (Gotelli, 2000). This algorithm has a low chance of falsely rejecting the null hypothesis (Type I error) (Gotelli, 2000). To illustrate species distribution in our study area, we performed a logistic regression model using species relative frequencies as response data and distance to the perimeter of the rice fields as spatial gradient.

The relationships between the species pattern and the environmental variables measured in the field were analysed using the multivariate CANOCO Version 4.5 procedure and the results were visualized with the extension CanoDraw for Windows (Ter Braak \& Smilaurer, 2002). This multivariate method allows the identification of a subset of environmental parameters that best correlate to the biotic structure and thus may be assumed to strongly affect species patterns. Nominal variables were defined by series of indicators or dummy variables (one per category). A dummy variable takes the value of 1 when the sample belongs to a given category and 0 otherwise. Prior to analysis, species data were log-transformed. Principal component analysis (PCA) was first used to describe the general patterns of variance in species data. This analysis did not include any explanatory variable, but instead included passive variables. The distribution of the variability in species was visually checked to assess the existence of any pattern. Then, inferential analyses were completed using redundancy analysis (RDA) to estimate how much variation in the species data was attributable to environmental variables. The forwardselection option was used to determine the minimal set of environmental parameters required to explain the largest amount of variation in species data. At each step, the statistical significance of the environmental variable added in the course of the forward selection was tested by means of unrestricted Monte Carlo permutation test $(n=499)$. Then, a partial RDA analysis was performed with spatial and seasonal effects as covariates in order to identify other significant environmental factors that might be hidden by space and time effects.

\section{Results}

Species co-occurrence

Among 960 traps set during the survey, 125 were positive for emerging An. gambiae s.l. mosquitoes. Some of the putative larval habitats covered were negative throughout the survey. In total, 1364 An. gambiae s.l. were collected (591 males, 773 females) and 826 were identified by PCR ( 828 specimens were tested, of which two repeatedly failed to amplify). Overall, the $\mathrm{M}$ form accounted for $31 \%$ of the specimens collected, the S form for $55 \%$ and An. arabiensis for $14 \%$. One putative hybrid specimen between the $\mathrm{M}$ form and An. arabiensis was recorded and no MS hybrid pattern was observed. Species co-occurrence analysis indicated significant differences between the $\mathrm{C}$-scores $\left(\mathrm{C}\right.$-score Obs $>\mathrm{C}$-score Est $_{\text {; }}$; $P=0.042$ ), suggesting that members of the An. gambiae complex were not randomly distributed in the area. Logistic plotting using distance to the rice fields' perimeter as a spatial explanatory factor showed that the three taxa were distributed in relation to distance to the rice fields (Fig. 2). The M form was mainly found within the irrigated perimeter, where it represented $99 \%$ of emerging mosquitoes, whereas the $\mathrm{S}$ form and An. arabiensis were found in the surrounding areas.

\section{Gradient analysis}

Principal component analysis was used to analyse the distribution and mutual relationships between taxa and samples. The graphic representation shows an association between An. gambiae $\mathrm{S}$ form and An. arabiensis, which suggests that these species have similar ecological requirements (Fig. 3A). This group mapped together with quarries on the right side of the first axis and was located in savannah and village areas far from the rice fields. This is also the area of the graph in which the last months of the rainy season, September and October, are found. The $\mathrm{M}$ form is independent of the first axis and mainly correlates with the second axis. It mapped in the upper part of the ordination diagram, which represents complex habitats such as wide rice paddies with predators and Culex larvae. The first two axes of the ordination diagram explain $86.5 \%$ of the variability in species data.

Redundancy analysis showed that mosquito taxa segregate mainly along the first two axes, which accounted for $32.8 \%$ and $7.4 \%$ of the variance in species data, respectively, and $73.6 \%$ and $16.7 \%$ of the species-environment correlation, respectively (Fig. 3B). The correlation between species and environmental variables along these axes is high $\left(r^{2}=0.78\right)$ for the first axis and moderate $\left(r^{2}=0.49\right)$ for the second axis. The first axis defines a spatial gradient on which species segregated from the core of the rice field area to the surrounding villages and savannah areas. This gradient supports distinct ecosystems. Positive values on the axis represent complex and permanent habitats that consisted of rice fields with a large surface colonized by floating plants, contained predators and were found far from human dwellings. Negative values represent sites found far from the rice field area in surrounding savannah and villages in which mosquitoes 


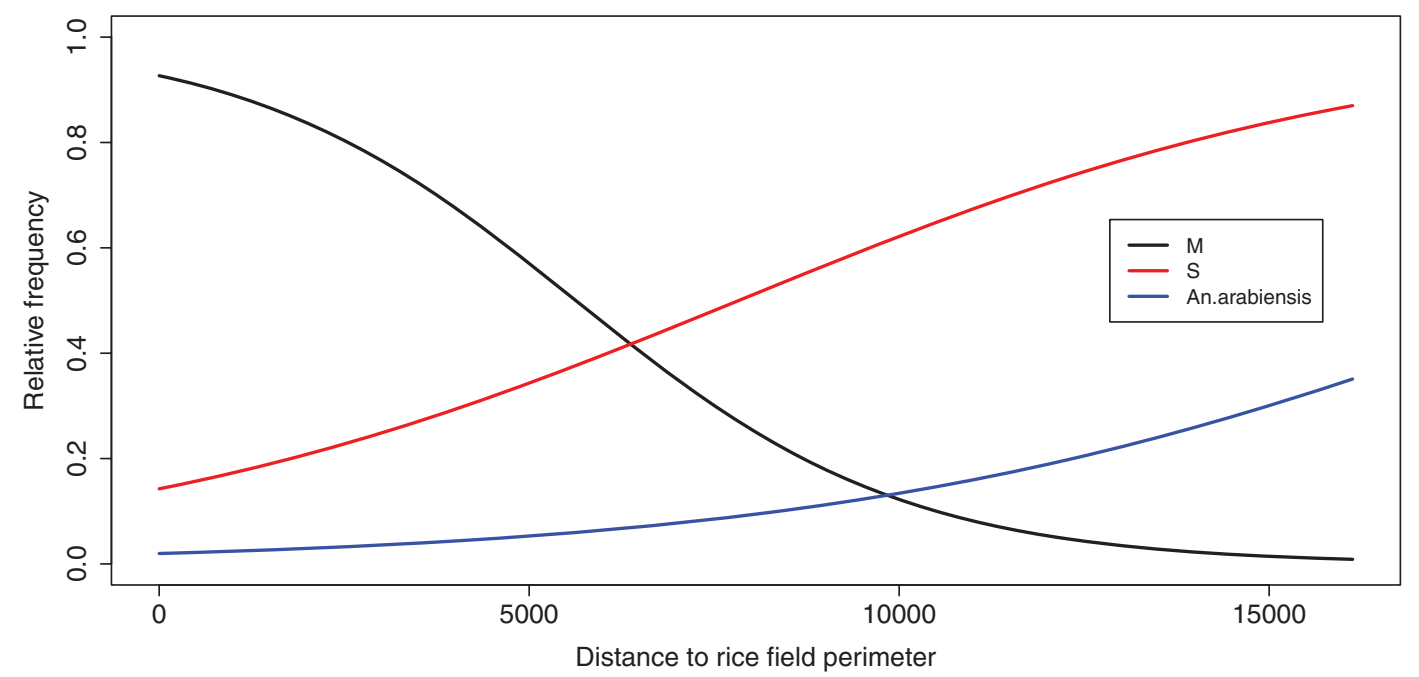

Fig. 2. Logistic plot of relative frequencies of the $\mathrm{M}$ and $\mathrm{S}$ forms of Anopheles gambiae and Anopheles arabiensis in relation to distance from the rice fields' perimeter.

exploited quarries. Negative values on the second axis are influenced by stream pools and floating plants and positive values are influenced by debris. The $\mathrm{M}$ form was associated with the early months (June to August) of the rainy season, whereas the $\mathrm{S}$ form and An. arabiensis were associated with the later months (September, October).

Six variables were significantly associated with mosquito distribution: distance to the rice fields' perimeter $(P=0.002)$; presence of floating plants $(P=0.002)$; culture $(P=0.048)$; distance to the nearest house $(P=0.012)$, rice field habitat ( $P=0.046)$, and month of October $(P=0.006)$. The rice field habitat, presence of floating plants, culture and distance to the nearest house were the best predictors of the $\mathrm{M}$ form larval habitat, whereas distance to the rice fields' perimeter and late collection month (October) appeared to be predictors for the $\mathrm{S}$ form and An. arabiensis.

Partial RDA (with collection month and distance to the rice fields considered as covariates) highlighted the additional effects of other explanatory variables potentially hidden by the main effects of distance to the rice fields and collection month. Analyses showed that mosquito taxa segregated along the first two axes, which accounted for $12.9 \%$ and $4.9 \%$ of the variance in species data, respectively, and for $68.5 \%$ and $25.8 \%$ of the species-environment correlation, respectively (Fig. 3C). This strong reduction in the proportion of total variance explained by environmental factors highlights the major effects of collection month and distance to the rice fields on mosquito distribution in our study area. The correlations between species and environmental variables along the first and second axes were moderate $\left(r^{2}=0.47\right.$ and $r^{2}=0.46$, respectively). Again, the first axis reflects a habitat complexity gradient. Positive values represent complex aquatic ecosystems, constituted by rice fields and stream pools that support high densities of predators, algae and floating plants and are found far from human dwellings. Negative values represent quarries found in savannah areas that seem to be less complex habitats in terms of the communities they support. The second axis could be considered as an anthropogenic gradient. Positive values represent environmental variables that characterize habitat degradation, such as the presence of debris, villages and ruts. Negative values represent more rural and feral savannah areas. The $\mathrm{M}$ form and An. arabiensis clustered along positive values on the second axis, which suggests that both taxa are more adapted to disturbed habitats, unlike the $\mathrm{S}$ form, which appears to be a savannah mosquito exploiting habitats with simple community structures. Two significant variables explained taxa distribution: floating plants $(P=0.004)$, and culture $(P=$ $0.034)$, mainly negatively correlated to the $\mathrm{S}$ form.

\section{Discussion}

According to Hutchinson (1978), in order to coexist, two species must differ to some extent in their ecological niche requirements or in some aspects of their life histories. Our survey showed that, at a microgeographic scale, the three sympatric taxa of the An. gambiae complex are not randomly distributed among aquatic habitats and that distance to the rice fields' perimeter appears as a structuring abiotic factor shaping their ecological niches. Furthermore, multifactorial analyses showed that the complexity of communities' arrangements in aquatic ecosystems is a key ecological factor modulating niche partitioning between these taxa.

Because community structure across freshwater habitat gradients is known to depend mainly upon physical factors, such as pond drying, and biotic effects mediated by ecological interactions, such as predation and competition (Wellborn et al., 1996), our study was based on the collection of mosquitoes emerging from larval development sites. Emergence is the endpoint of mosquito larval development. Our approach therefore has the advantage of providing a picture of the mosquito populations that successfully develop and emerge under different and specific selection pressures in various larval habitats. The relevance of this sampling method is further reinforced by 

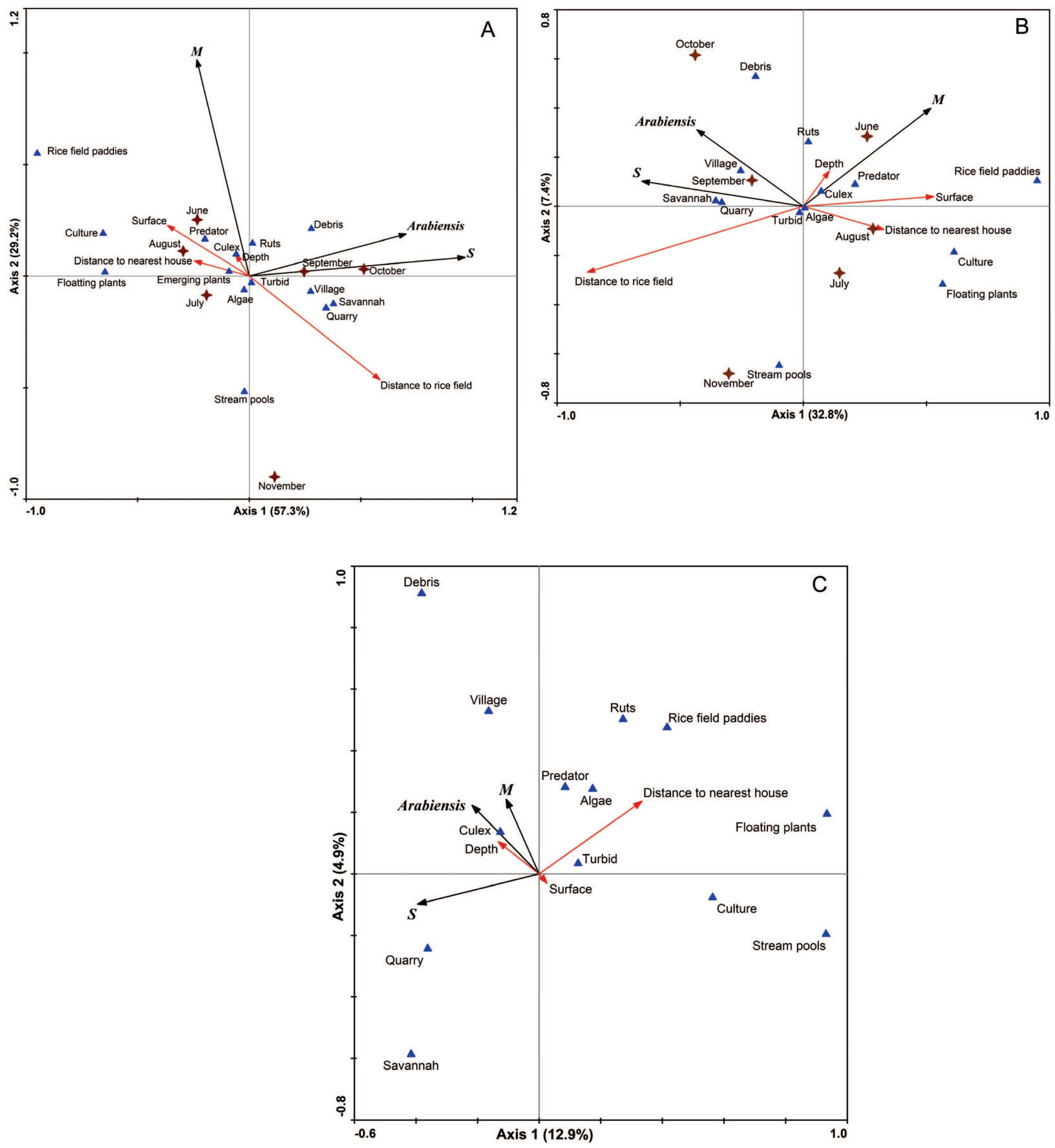

Fig. 3. Multifactorial analysis of the distribution of Anopheles gambiae complex mosquitoes in relation to environmental variables. (A) A principal component analysis diagram shows passive plotting of environmental variables. (B) Redundancy analysis (RDA) ordination diagram. (C) Partial RDA species-environment biplot. On each graph the proportion of total variance in species data explained by each axis is given in brackets. Blue triangles represent nominal environmental factors and red arrows represent continuous environmental factors. Mosquito taxa are represented by black arrows and collection months by brown crosses.

recent studies demonstrating different levels of susceptibility to predation between molecular forms of An. gambiae (Diabaté et al., 2008; Gimonneau et al., 2010). One drawback, however, is that sample sizes are lower than they would be if larval immature specimens were directly collected from breeding sites in terms of both the number of positive collections (i.e. the number of collections in which at least one adult An. gambiae specimen is present) and the number of specimens collected per sampling unit. This obviously calls for increased sampling effort in order to gain statistical power. However, the fact that 
only 125 of 960 traps (i.e. 13\%) were positive for emerging An. gambiae s.l. mosquitoes is unlikely to have biased our results and inferences because all taxa should be exposed to the same probability of sampling upon emergence. Variations in egg hatching time and larval development time within and between species and molecular forms in the An. gambiae complex as well as across water types (Diabaté et al., 2005, 2008; Yaro et al., 2006) result in the distribution of emerging mosquitoes across several days, ensuring representative sampling of emerging mosquitoes throughout a 24-h period.

Principal component analysis identified a pattern of association between the $\mathrm{S}$ form and An. arabiensis, whereas the $\mathrm{M}$ form was independent of this group. A high proportion of species variance was explained along the first two axes (86.5\%). The $\mathrm{S}$ form and An. arabiensis clustered on the first axis, indicating that these taxa seem to segregate in relation to distance to the rice fields' perimeter. Conversely, the $\mathrm{M}$ form was mainly associated with the second axis, which negatively correlated with distance to the rice fields and, as a consequence, positively correlated with rice field habitat. This suggests that the spatial pattern represented by the distance to the rice fields' perimeter acts as a major environmental factor in structuring the ecological niches of members of the An. gambiae complex.

This result was confirmed by RDA. Distance to the rice fields' perimeter was the most significant factor along which species segregated. Other significant environmental factors representative of the irrigated perimeter were highlighted by RDA, such as the presence of floating plants, distance to the nearest house, culture and rice field habitat. These key environmental parameters were all associated with the presence of the $\mathrm{M}$ form. The significant association with floating plants (mainly Marsilea polycarpa and Nymphea sp.) is certainly the most important ecological factor as it could be considered a biomarker of habitat stability and, more generally, of habitat complexity because plants offer refuges that allow many species to escape predation (Sih, 1987). Habitat complexity can be illustrated by the presence of predators (mainly Notonectidae and Dytiscidae) and Culex larvae (mainly Cx. quinquefasciatus), which were found in high densities in the rice paddies. The high diversity found in this type of habitat (Roger, 1996; Bambaradeniya et al., 2004) favours and sustains, in turn, complex trophic relationships and multiple biological interactions between species (Menge \& Sutherland, 1976).

Temporal pattern was significant in October and associated with the presence of the $\mathrm{S}$ form and An. arabiensis, which were found to exploit the same breeding sites at the end of the rainy season. This reflects a population turnover during the late rainy season (Diabaté et al., 2002), when 51\% of all An. arabiensis specimens were collected in October. This also suggests an overlap in ecological niches driven by the scarcity of temporary breeding sites available for female ovipositioning in savannah areas at the end of the rainy season. Although the $\mathrm{M}$ form was present throughout the year in the locality of Bama (Diabaté et al., 2002; Baldet et al., 2003), this mosquito was only associated with the early months (June-August) of the rainy season. This pattern may be linked to the rice cultivation cycle in that rice paddies are more suitable for An. gambiae development during the early phases of crop cultivation (Baldet et al., 2003).
The RDA ordination diagram also shows that the $\mathrm{S}$ form and An. arabiensis tend to exploit temporary pools in savannah areas and villages located far from the perimeter of the rice fields. Their preferred habitat types are generally vegetationfree $(73 \%$ and $85 \%$ without floating plants for the $\mathrm{S}$ form and An. arabiensis, respectively) indicating that these taxa exploit simple rain-dependent temporary aquatic habitats. This is consistent with previous observations in Mali, where the ecological niches of species seem to segregate according to habitat type and temporality (Edillo et al., 2002). Here, our results identified distance to the perimeter of the rice fields as the main physical factor modulating the distribution of species and molecular forms. However, this dimension also has an ecological value because these taxa segregated through a habitat complexity gradient supported by the hydro-periodicity of the larval habitat. As a result, distance to the rice fields' perimeter represents an ecological cline in which a range of breeding sites are represented by permanent and complex aquatic habitat at one end and by temporary and simpler habitat at the other. This ecological gradient, which extends from permanent to temporary freshwater habitats, has long been recognized as critical in shaping the structure of aquatic communities in relation to the ecological interactions they support (Schneider \& Frost, 1996; Wellborn et al., 1996).

As a result, the rice fields' perimeter appears to be the core habitat of the $\mathrm{M}$ form, from which it successfully emerges and spreads into the surrounding savannahs and villages in which the $\mathrm{S}$ form and An. arabiensis thrive. This result confirms previous studies showing that the $\mathrm{M}$ form outcompetes the $\mathrm{S}$ form in permanent aquatic habitats (Diabaté et al., 2008). Predation pressure is more important in permanent than in temporary habitats (Sunahara et al., 2002; Diabaté et al., 2008) and was clearly identified as a biotic process that contributes to the ecological niche segregation between forms (Gimonneau et al., 2010). However, we might expect that An. arabiensis and the $\mathrm{M}$ form share part of their larval niche requirements, as in East Africa, where the $\mathrm{M}$ form is absent and An. arabiensis exploits rice paddies (Muturi et al., 2008). These differences in species distribution between East and West Africa probably reflect different evolutionary paths mediated by species interactions in these different geographical contexts. In West Africa, interaction with the $\mathrm{M}$ form in permanent habitats may have led to ecological niche displacement of An. arabiensis. Although competitive exclusion between these species has never been documented, this observation suggests that the $M$ form may be more competitive than its sibling species in this type of habitat in West Africa. Indeed, it is noticeable that populations of An. arabiensis, as well as those of the $\mathrm{S}$ form of An. gambiae, from West and East Africa (i.e. on both sides of the Great Rift Valley) are equipped with different arrangements of chromosomal inversions and might therefore represent distinct ecotypes populating distinct ecological niches in West and East Africa (Petrarca et al., 2000; Coluzzi et al., 2002; Pombi et al., 2008).

However, the $\mathrm{M}$ form and An. arabiensis seem well adapted to anthropogenic contexts as they segregated together along a human disturbance gradient in partial RDA. In East and West Africa, studies have shown that An. arabiensis can breed in rice paddies (Muturi et al., 2008) and urban areas (Robert 
et al., 1998; Jacob et al., 2003; Machault et al., 2009). In Burkina Faso, both species have recently been found in periurban and urban areas in polluted larval habitats (Fournet $e t$ al., 2010). Moreover, the association with Culex larvae, which are commonly found in highly polluted aquatic habitats (Subra, 1981), reflects the anthropogenic nature of these breeding sites. These findings are in agreement with our results and suggest that An. gambiae $\mathrm{M}$ form and An. arabiensis are adapting to anthropogenic contexts. By contrast, the $\mathrm{S}$ form appears to be a more feral mosquito that is mainly found in savannah areas and is able to exploit quarries.

The fact that most species living in temporary habitats are absent from permanent ones (Wiggins et al., 1980) suggests that some factors such as predation and/or competition may prevent species from occupying habitats that are apparently suitable to their development. Susceptibility to predation has been shown to be one of the main forces structuring species among aquatic habitats (Wellborn et al., 1996). The M form, which is totally dependent on permanent habitats during the dry season, was shown to demonstrate predator-avoidance behaviour in predator-rich habitats (Gimonneau et al., 2010). By contrast, more rapid larval development, as observed in the S form (Diabaté et al., 2005, 2008; Lehmann \& Diabaté, 2008), represents better adaptation to fluctuating habitats with a high risk of desiccation. Consequently, fitness success at one end of the gradient implies lower performance at other end, which results in the observed pattern of distribution of members of the An. gambiae complex.

This study allowed us to better understand the ecological requirements of sympatric taxa of the An. gambiae complex unevenly distributed among aquatic habitats in a savannah area in southern Burkina Faso. Species and molecular forms were sharply segregated along a gradient ranging from 'permanent anthropic' habitats, exploited by the $\mathrm{M}$ form, to 'temporary natural' habitats, in which the $\mathrm{S}$ form and An. arabiensis are found. Moreover, An. arabiensis is more strongly associated with village areas, which suggests that, like the $\mathrm{M}$ form, it is more adapted to anthropogenic conditions. Consequently, the larval ecology of this species complex appears to be clearly linked to the hydro-periodicity of habitats and to the ecological communities they support in relation to anthropogenic activities. In the actual context of dam building for irrigated agriculture in rural and peri-urban areas in West Africa as part of the drive to increase food self-sufficiency, understanding the distribution of vector species gains value in the prevention of malaria transmission in these new modified environments.

\section{Acknowledgements}

We thank Baudoin Dabiré, Boubacar Nikiema, Hervé Somda and Pascal Yéyé for excellent assistance in field and laboratory work. We also thank Phillipe Cecchi, Carlo Costantini and Jérémy Bouyer for constructive discussions, and Joost Wellens for GIS assistance.

This work was supported in parts by a National Institutes of Health grant (R01-A1063508) to F. S. under the leadership of N. J. Besansky (Notre Dame University, Notre Dame, IN, U.S.A.) and by the French National Research Agency under the reference ANR-08-MIEN-006 to FS. GG was supported by a $\mathrm{PhD}$ fellowship from the Fondation pour la Recherche Médicale (FRM).

\section{References}

Baldet, T., Diabaté, A. \& Guiguemde, T.R. (2003) Malaria transmission in 1999 in the rice field area of the Kou Valley (Bama), (Burkina Faso). Santé, 13, 55-60.

Bambaradeniya, C.N.B., Edirisinghe, J.P., De Silva, D.N., Gunatilleke, C.V.S., Ranawana, K.B. \& Wijekoon, S. (2004) Biodiversity associated with an irrigated rice agro-ecosystem in Sri Lanka. Biodiversity and Conservation, 13, 1715-1753.

Clark, G.G., Seda, H. \& Gubler, D.J. (1994) Use of the CDC backpack aspirator for surveillance of Aedes aegypti in San Juan, Puerto Rico. Journal of the American Mosquito Control Association, 10, 119-124.

Coluzzi, M. (1999) The clay feet of the malaria giant and its African roots: hypotheses and inferences about origin, spread and control of Plasmodium falciparum. Parassitologia (Rome), 41, 277-283.

Coluzzi, M., Petrarca, V. \& Di deco, M.A. (1985) Chromosomal inversion intergradation and incipient speciation in Anopheles gambiae. Bollettino di Zoologia, 52, 45-63.

Coluzzi, M., Sabatini, A., della Torre, A., Di Deco, M.A. \& Petrarca, V. (2002) A polytene chromosome analysis of the Anopheles gambiae species complex. Science, 298, 1415-1418.

Connor, E.F. \& Simberloff, D. (1979) The assembly of species communities: chance or competition. Ecology, 60, 1132-1140.

Costantini, C., Ayala, D., Guelbeogo, W. et al. (2009) Living at the edge: biogeographic patterns of habitat segregation conform to speciation by niche expansion in Anopheles gambiae. BMC Ecology, 9, 16.

della Torre, A., Fanello, C., Akogbeto, M. et al. (2001) Molecular evidence of incipient speciation within Anopheles gambiae s.s. in West Africa. Insect Molecular Biology, 10, 9-18.

della Torre, A., Tu, Z. \& Petrarca, V. (2005) On the distribution and genetic differentiation of Anopheles gambiae s.s. molecular forms. Insect Biochemistry and Molecular Biology, 35, 755-759.

Diabaté, A., Baldet, T., Chandre, F. et al. (2002) The role of agricultural use of insecticides in resistance to pyrethroids in Anopheles gambiae s.l. in Burkina Faso. American Journal of Tropical Medicine and Hygiene, 67, 617-622.

Diabaté, A., Dabiré, R.K., Kim, E.H. et al. (2005) Larval development of the molecular forms of Anopheles gambiae (Diptera: Culicidae) in different habitats: a transplantation experiment. Journal of Medical Entomology, 42, 548-553.

Diabaté, A., Dabiré, R., Heidenberger, K. et al. (2008) Evidence for divergent selection between the molecular forms of Anopheles gambiae: role of predation. BMC Evolutionary Biology, 8, 5.

Edillo, F.E., Toure, Y.T., Lanzaro, G.C., Dolo, G. \& Taylor, C.E. (2002) Spatial and habitat distribution of Anopheles gambiae and Anopheles arabiensis (Diptera: Culicidae) in Banambani Village, Mali. Journal of Medical Entomology, 39, 70-77.

Fontenille, D. \& Simard, F. (2004) Unravelling complexities in human malaria transmission dynamics in Africa through a comprehensive knowledge of vector populations. Comparative Immunology, Microbiology and Infectious Diseases, 27, 357-375.

Fournet, F., Cussac, M., Ouari, A. et al. (2010) Diversity in anopheline larval habitats and adult composition during the dry and wet seasons in Ouagadougou (Burkina Faso). Malaria Journal, 9, 78. 
Funk, D.J. (1998) Isolating a role for natural selection in speciation: host adaptation and sexual isolation in Neochlamisus bebbianae leaf beetles. Evolution, 52, 1744-1759.

Funk, D.J., Filchak, K.E. \& Feder, J.L. (2002) Herbivorous insects: model systems for the comparative study of speciation ecology. Genetica, 116, 251-267.

Gillies, M.T. \& De Meillon, B. (1968) The Anophelinae of Africa South of the Sahara (Ethiopian Zoogeographical Region). South African Institute for Medical Research, Johannesburg.

Gimonneau, G., Bouyer, J., Morand, S., Besansky, N.J., Diabate, A. \& Simard, F. (2010) A behavioural mechanism underlying ecological divergence in the malaria mosquito Anopheles gambiae. Behavioral Ecology, 21, 1087-1092.

Gotelli, N.J. (2000) Null model analysis of species co-occurrence patterns. Ecology, 81, 2606-2621.

Gotelli, N.J. \& Entsminger, G.L. (2010) EcoSim: Null Models Software for Ecology, Version 7. Acquired Intelligence, Inc. \& KeseyBear, Jericho, VT. http://garyentsminger.com/ecosim.htm [Accessed 18 May 2010].

Hutchinson, E.G. (1978) What is a niche? An Introduction to Population Ecology (ed. by E. G. Hutchinson), pp. 152-219. Yale University Press, London.

Jacob, B.G., Regens, J.L., Mbogo, C.M. et al. (2003) Occurrence and distribution of Anopheles (Diptera: Culicidae) larval habitats on land cover change sites in urban Kisumu and urban Malindi, Kenya. Journal of Medical Entomology, 40, 777-784.

Lawniczak, M.K.N., Emrich, S.J., Holloway, A.K. et al. (2010) Widespread divergence between incipient Anopheles gambiae species revealed by whole genome sequences. Science, 330, 512-514.

Lefèvre, T., Gouagna, L.C., Dabiré, K.R. et al. (2009) Beyond nature and nurture: phenotypic plasticity in blood-feeding behaviour of Anopheles gambiae s.s. when humans are not readily accessible. American Journal of Tropical Medicine and Hygiene, 81, 1023-1029.

Lehmann, T. \& Diabaté, A. (2008) The molecular forms of Anopheles gambiae: a phenotypic perspective. Infection Genetics and Evolution, 8, 737-746.

Machault, V., Gadiaga, L., Vignolles, C. et al. (2009) Highly focused anopheline breeding sites and malaria transmission in Dakar. Malaria Journal, 8, 138.

Mallet, J. (2008) Hybridization, ecological races and the nature of species: empirical evidence for the ease of speciation. Philosophical Transactions of the Royal Society B: Biological Sciences, 363, 2971-2986.

Menge, B.A. \& Sutherland, J.P. (1976) Species diversity gradients: synthesis of roles of predation, competition, and temporal heterogeneity. American Naturalist, 110, 351-369.

Muturi, E.J., Muriu, S., Shililu, J. et al. (2008) Effect of rice cultivation on malaria transmission in central Kenya. American Journal of Tropical Medicine and Hygiene, 78, 270-275.

Neafsey, D.E., Lawniczak, M.K.N., Park, D.J. et al. (2010) SNP genotyping defines complex gene-flow boundaries among African malaria vector mosquitoes. Science, 330, 514-517.

Nosil, P., Harmon, L.J. \& Seehausen, O. (2009) Ecological explanations for (incomplete) speciation. Trends in Ecology and Evolution, 24, 145-156.

Palumbi, S.R. (2001) Evolution-humans as the world's greatest evolutionary force. Science, 293, 1786-1790.

Petrarca, V., Nugud, A.D., Ahmed, M.A.E., Haridi, A.M., Di Deco, M.A. \& Coluzzi, M. (2000) Cytogenetics of the Anopheles gambiae complex in Sudan, with special reference to An. arabiensis: relationships with East and West African populations. Medical and Veterinary Entomology, 14, 149-164.
Pombi, M., Caputo, B., Simard, F. et al. (2008) Chromosomal plasticity and evolutionary potential in the malaria vector Anopheles gambiae sensu stricto: insights from three decades of rare paracentric inversions. BMC Evolutionary Biology, 8, 309.

Rice, W.R. \& Hostert, E.E. (1993) Laboratory experiments on speciation: what have we learned in 40 years? Evolution, 47, 1637-1653.

Robert, V., Awono-Ambene, H.P. \& Thioulouse, J. (1998) Ecology of larval mosquitoes, with special reference to Anopheles arabiensis (Diptera: Culicidae) in market-garden wells in urban Dakar, Senegal. Journal of Medical Entomology, 35, 948-955.

Roger, P.A. (1996) Biology and Management of the Floodwater Ecosystem in Ricefields. ORSTOM, Paris.

Santolamazza, F., Mancini, E., Simard, F., Qi, Y., Tu, Z. \& della Torre, A. (2008) Insertion polymorphisms of SINE200 retrotransposons within speciation islands of Anopheles gambiae molecular forms. Malaria Journal, 7, 163.

Schluter, D. (2001) Ecology and the origin of species. Trends in Ecology and Evolution, 16, 372-380.

Schluter, D. (2009) Evidence for ecological speciation and its alternative. Science, 323, 737-741.

Schneider, D.W. \& Frost, T.M. (1996) Habitat duration and community structure in temporary ponds. Journal of the North American Benthological Society, 15, 64-86.

Sih, A. (1987) Prey refuges and predator prey stability. Theoretical Population Biology, 31, 1-12.

Simard, F., Ayala, D., Kamdem, G. et al. (2009) Ecological niche partitioning between Anopheles gambiae molecular forms in Cameroon: the ecological side of speciation. BMC Ecology, 9, 17.

Stone, L. \& Roberts, A. (1990) The checkerboard score and species distributions. Oecologia, 85, 74-79.

Subra, R. (1981) Biology and control of Culex pipiens quinquefasciatus Say, 1823 (Diptera, Culicidae) with special reference to Africa. Insect Science and its Application, 1, 319-338.

Sunahara, T., Ishizaka, K. \& Mogi, M. (2002) Habitat size: a factor determining the opportunity for encounters between mosquito larvae and aquatic predators. Journal of Vector Ecology, 27, 8-20.

Ter Braak, C.J.F. \& Smilaurer, P. (2002) CANOCO Reference Manual and Cano Draw for Windows User's Guide: Software for Canonical Community Ordination (Version 4.5). Microcomputer Power, New York, NY.

Touré, Y.T., Petrarca, V., Traore, S.F. et al. (1994) Ecological genetic studies in the chromosomal form Mopti of Anopheles gambiae s.str. in Mali, West Africa. Genetica, 94, 213-223.

Wellborn, G.A., Skelly, D.K. \& Werner, E.E. (1996) Mechanisms creating community structure across a freshwater habitat gradient. Annual Review of Ecology and Systematics, 27, 337-363.

Wellens, J., Diallo, M., Dakouré, D., Compaoré, N.F., Denis, A. \& Tychon, B. (2008) Utilisation des prises de vue aérienne à basse altitude pour le suivi des activités hydro-agricoles-Cas du Bassin du Kou. Proceedings of the Salon Africain d'Irrigation et Drainage, Ouagadougou, Burkina Faso, 29-30 January 2008.

White, B.J., Cheng, C.D., Simard, F., Costantini, C. \& Besansky, N.J. (2010) Genetic association of physically unlinked islands of genomic divergence in incipient species of Anopheles gambiae. Molecular Ecology, 19, 925-939.

Wiggins, G.B., Mackay, R.J. \& Smith, I.M. (1980) Evolutionary and ecological strategies of animals in annual temporary ponds. Archiv fur Hydrobiologie: Supplement, 58, 97-206.

Yaro, A.S., Dao, A., Adamou, A. et al. (2006) The distribution of hatching time in Anopheles gambiae. Malaria Journal, 5, 19.

Accepted 10 February 2011 